\title{
Chemical Rocket Propulsion
}

\author{
David Greatrix, ${ }^{1}$ Ivett Leyva, ${ }^{2}$ Dario Pastrone, ${ }^{3}$ \\ Valsalayam Sanal Kumar, ${ }^{4}$ and Michael Smart ${ }^{5}$ \\ ${ }^{1}$ Department of Aerospace Engineering, Ryerson University, Toronto, ON, Canada M5B 2K3 \\ ${ }^{2}$ Aerophysics Branch, Air Force Research Laboratory, Edwards AFB, CA 93524, USA \\ ${ }^{3}$ Dipartimento di Ingegneria Meccanica e Aerospaziale, Politecnico di Torino, 10129 Torino, Italy \\ ${ }^{4}$ Department of Aeronautical Engineering, Kumaraguru College of Technology, Coimbatore, Tamil Nadu 641006, India \\ ${ }^{5}$ School of Mechanical and Mining Engineering, The University of Queensland, Brisbane, QLD 4072, Australia
}

Correspondence should be addressed to David Greatrix, greatrix@ryerson.ca

Received 31 October 2012; Accepted 31 October 2012

Copyright (c) 2012 David Greatrix et al. This is an open access article distributed under the Creative Commons Attribution License, which permits unrestricted use, distribution, and reproduction in any medium, provided the original work is properly cited.

We are pleased to present to you this special issue on chemical rocket propulsion. It is hoped that experts and nonexperts alike will enjoy the discussion of a number of international research efforts that are taking place across the breadth of this diverse field, as conveyed by the authors of the papers appearing herein. The submitted papers certainly reveal the wide number of disciplines (chemistry, fluid dynamics, structures, etc.) that currently play important roles towards ultimately producing effective chemical rocket systems.

Recently, an important milestone has been reached in the history of chemical rocket propulsion, with the retirement of the Space Shuttle. The end of one era brings the dawn of a new era in space transportation, with the anticipation that, with time, new and better flight vehicles will come on the scene and flourish in their respective applications. Almost surely, those new vehicles will still be propelled in large part by chemical rocket systems, systems that have been updated and improved over those of the previous generation through the efforts of today's researchers and engineers.

Liquid-propellant rocket engines continue as the preeminent chemical rocket propulsion system, from millinewton spacecraft thrusters to meganewton first-stage engines for space launch vehicles. Although it is commonly perceived that liquid rocket engines are a mature technology, there are still many active research areas. For example, replacing highly toxic and expensive-to-handle propellants used in hypergolic systems with greener, less toxic propellants is a current challenge. As the thrust demands go up, achieving longer life for monopropellant systems especially remains an objective. For missions beyond Earth's orbit, proven relight capability for upper stage engines, and long-term storage for cryogens, will become some of the engineering issues researchers will face. Liquid propulsion will remain a critical component in launch systems in the foreseeable future. In conjunction, the interest in making these systems more reliable, more durable, and less expensive to develop and acquire for a number of diverse flight mission applications will continue, and the engineering challenges associated with these objectives will have to be met.

Solid-propellant rocket motors maintain their importance in meeting the propulsion needs for a number of flight applications, big and small. While solid rockets have had, for a long time now, the reputation as being the cost-effective, ready-to-go option, research continues on improving all aspects of their performance, including their safety and friendliness to the surrounding environment. For example, ammonium dinitramide (ADN) appears to be making inroads as a potential greener replacement for ammonium perchlorate (AP) as an effective oxidizer for a number of solid propellants. There is some interest in going to higher chamber pressures to increase thrust-related performance; at higher pressures, one may encounter the need to more actively inhibit the appearance of combustion instability symptoms during a given motor's operation.

Manned suborbital flights powered by hybrid rocket engines have recently become reality, mainly due to reasons related to low cost and safety. Potential applications of hybrid rockets range from microgravity platforms to launchers and landing vehicles, but researchers must face some challenges, in part due to the peculiar combustion process of hybrid 
propellants. Different concepts are being studied as a means to increase grain regression rate, from innovative fuels (e.g., paraffin-based solid fuels or solid methane) to innovative engine architectures (e.g., advanced vortex-hybrid or cascaded multistage impinging-jet designs). Other important issues, which must be dealt with, are mixture ratio shifting, combustion efficiency, and combustion instability.

Air-breathing rocket engines continue to be a practical propulsion system candidate for some smaller, longer-range, and high-speed missile applications. Challenges remain moving up in scale and scope, for military and civil applications such as single-stage-to-orbit flight vehicles. International computational and experimental research efforts continue in this regard.

Within the aerospace propulsion community, one commonly hears that every significant advance in aerospace transportation has been made possible by a significant advance in propulsion technology. One also hears that, as engineers and scientists, when we are fortunate enough to make significant progress in our time, we do so with the realization that we stand on the shoulders of giants. The giants, of course, are those who paved the way before us, with their insight, perseverance, and skill. This is certainly true of the field of chemical rocket propulsion. With this in mind, one can say, with some confidence, that despite, the inevitable challenges to come, the future of chemical rocket propulsion is bright.

David Greatrix Ivett Leyva Dario Pastrone Valsalayam Sanal Kumar Michael Smart 

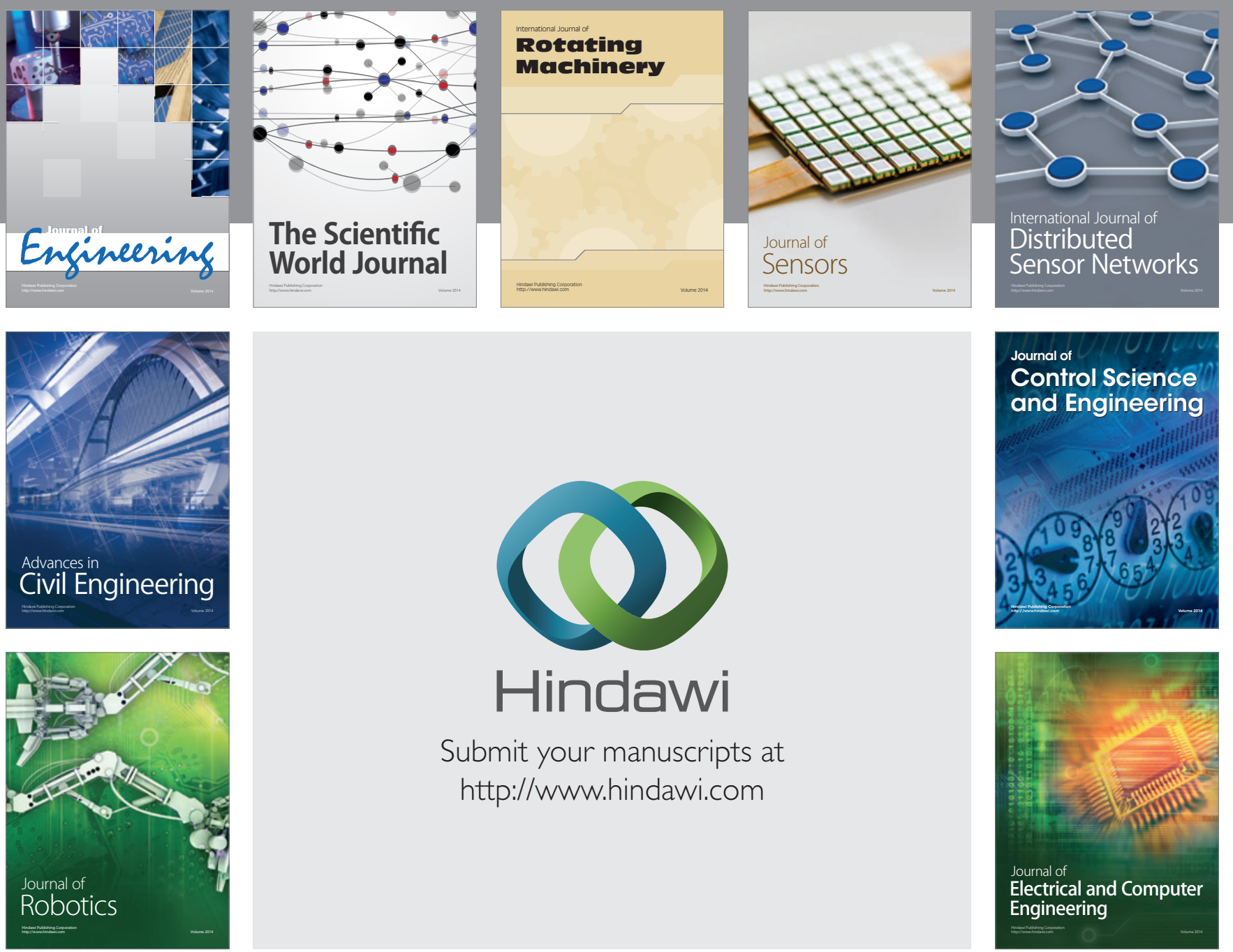

Submit your manuscripts at

http://www.hindawi.com
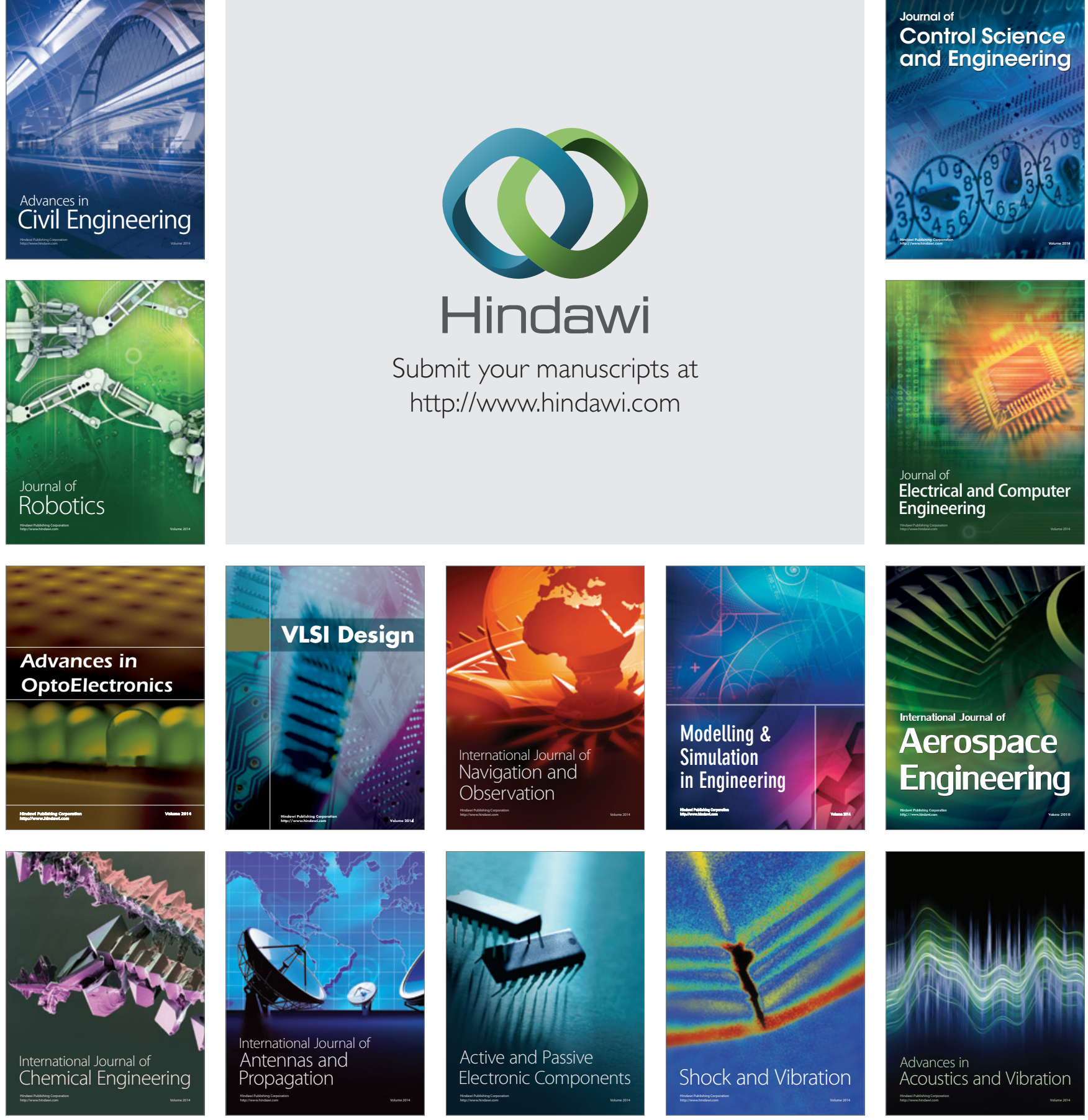